\title{
Inhaled and intranasal ciclesonide for the treatment of covid-19 in adult outpatients: CONTAIN phase II randomised controlled trial
}

\author{
Nicole Ezer, ${ }^{1,2}$ Sara Belga, ${ }^{3}$ Nick Daneman, ${ }^{4}$ Adrienne Chan, ${ }^{4}$ Benjamin M Smith, ${ }^{1,2}$ \\ Shay-Anne Daniels, ${ }^{3}$ Kristen Moran, ${ }^{5}$ Charlotte Besson, ${ }^{2}$ Louisa Y Smyth, ${ }^{5}$ Susan J Bartlett, ${ }^{2,6}$ \\ Andrea Benedetti, ${ }^{2,6}$ James G Martin, ${ }^{1,2}$ Todd C Lee, ${ }^{2,5,6,7}$ Emily G McDonald ${ }^{2,5,6,8}$
}

\section{ABSTRACT}

OBJECTIVE

To determine if inhaled and intranasal ciclesonide are superior to placebo at decreasing respiratory symptoms in adult outpatients with covid-19.

\section{DESIGN}

Randomised, double blind, placebo controlled trial.

SETTING

Three Canadian provinces (Quebec, Ontario, and British Columbia).

PARTICIPANTS

203 adults aged 18 years and older with polymerase chain reaction confirmed covid-19, presenting with fever, cough, or dyspnoea.

\section{INTERVENTION}

Participants were randomised to receive either inhaled ciclesonide $(600 \mu \mathrm{g}$ twice daily) and intranasal ciclesonide (200 $\mu$ g daily) or metered dose inhaler and nasal saline placebos for 14 days.

\section{MAIN OUTCOME MEASURES}

The primary outcome was symptom resolution at day 7 . Analyses were conducted on the modified intention-to-treat population (participants who took at least one dose of study drug and completed one follow-up survey) and adjusted for stratified randomisation by sex.

RESULTS

The modified intention-to-treat population included 203 participants: 105 were randomly assigned to ciclesonide (excluding two dropouts and one loss to follow-up) and 98 to placebo (excluding three dropouts and six losses to follow-up). The median age was 35 years (interquartile range 27-47 years) and $54 \%$ were women. The proportion of

\section{WHAT IS ALREADY KNOWN ON THIS TOPIC}

Treatments for adult outpatients with covid-19 are urgently needed

Recent clinical trials have shown efficacy of steroids in patients admitted to hospital with respiratory symptoms who require oxygen

Only two other trials of inhaled steroids suggest an impact on earlier resolution of symptoms in adult outpatients; however, those trials were non-placebo controlled

\section{WHAT THIS STUDY ADDS}

Compared with placebo, the combination of inhaled and intranasal ciclesonide was not associated with an appreciable increase in symptom resolution among healthier young adults with covid-19 who presented with cough, dyspnoea, or fever participants with resolution of symptoms by day 7 did not differ significantly between the intervention group (42/105, 40\%) and control group (34/98, $35 \%)$; absolute adjusted risk difference 5.5\% (95\% confidence interval $-7.8 \%$ to $18.8 \%$ ). Results might be limited to the population studied, which mainly included younger adults without comorbidities. The trial was stopped early, therefore could have been underpowered.

\section{CONCLUSION}

Compared with placebo, the combination of inhaled and intranasal ciclesonide did not show a statistically significant increase in resolution of symptoms among healthier young adults with covid-19 presenting with prominent respiratory symptoms. As evidence is insufficient to determine the benefit of inhaled and intranasal corticosteroids in the treatment of covid-19, further research is needed.

TRIAL REGISTRATION

ClinicalTrials.gov NCT04435795.

\section{Introduction}

In 2020, SARS-CoV-2 rapidly emerged in a global pandemic. Complications from covid-19 range from mild to severe, with some people remaining relatively asymptomatic and others requiring hospital admission, intubation, and critical care. Although several successful treatments have been identified for patients with covid-19 admitted to hospital, including dexamethasone, ${ }^{1}$ heparin, ${ }^{2}$ and tocilizumab, ${ }^{3}$ treatment options for outpatients have lagged. Although combination monoclonal antibody therapy seems to decrease rates of medically attended visits in higher risk patients, ${ }^{4}$ it is costly, not universally available, and, unlike oral and inhaled treatments, is complex to administer. Hence, the standard of outpatient care for most healthy adults remains supportive measures and quarantine. Nonetheless, infected patients can have a substantial symptom burden from cough and dyspnoea, and a subset might go on to require hospital admission. A safe, inexpensive treatment that can be administered at home and that reduces symptom burden and hospital admission is still urgently needed.

In February 2020, 5406 compounds, including 2069 US Food and Drug Administration approved drugs, were tested for antibodies to Middle East respiratory syndrome related coronavirus by determining viral spike protein expression in infected Vero cells. ${ }^{5}$ Twelve compounds were selected that had shown $>70 \%$ activity to antibodies against Middle East respiratory 
syndrome related coronavirus. ${ }^{5}$ Ciclesonide, a corticosteroid used in inhaled form to treat asthma and intranasally to treat allergic rhinitis, was identified as one of those drugs. In addition to its intrinsic antiinflammatory function, ciclesonide showed a potent antiviral effect when specifically screened for activity against SARS-CoV-2. ${ }^{6}$ On the basis of in vitro data and the known anti-inflammatory effect of ciclesonide, we hypothesised that ciclesonide administered early in the course of covid-19 could decrease symptom burden in adults presenting with prominent respiratory symptoms.

\section{Methods \\ Study design}

We conducted a randomised, double blind, placebo controlled trial (Inhaled Ciclesonide for the Treatment of COVID-19 in Nonhospitalized Adults (CONTAIN)) to evaluate the resolution of symptoms in adults with covid-19 presenting with predominantly respiratory symptoms. Participants were randomly assigned in a $1: 1$ ratio to receive either inhaled and intranasal ciclesonide or inhaled and intranasal placebo. Investigators, participants, and statisticians were blinded to treatment allocation. In the province of Quebec, recruitment occurred primarily through social media and by telephone contact of people who had tested positive for SARS-Cov-2 at the McGill University Health Centre. In Ontario, participants were recruited from a virtual covid-19 telehealth clinic at Sunnybrook Health Sciences Centre. In British Columbia, one health authority provided access to its regional list of people who had tested positive for SARS-CoV-2 and they were contacted and recruited by telephone. Participants were recruited province-wide from across Quebec, from the catchment area serviced by Sunnybrook Health Science Centre in Ontario and from the Vancouver Coastal Health region in British Columbia.

Participants self-screened and, if eligible, enrolled through a secure internet based survey using the Research Electronic Data Capture system. Participants provided an electronic signature after reading a consent form online and answering a series of comprehension questions. A survey was completed on the day of enrolment, on day 0 (the day treatment was received, before initiation), and on days 1, 3, 7, 10 , and 14. A follow-up survey was sent on day 29 to capture longer term outcomes (reported separately). Participants were sent reminder emails if they did not complete surveys, or they were contacted by telephone to capture outcomes. All participants provided an emergency contact number in the event they did not respond to email or telephone calls. The emergency contact number was used (maximum 10 attempts) to determine if failure to respond to emails and phone calls was secondary to illness or death.

We hypothesised that treatment would be most effective if given early in the disease process. As such, we aimed to recruit participants within five days of a positive polymerase chain reaction test result for SARS-CoV-2 and symptom onset.

\section{Protocol changes and rationale}

Because of the evolving covid-19 pandemic and the rapidity of new information available to investigators, we made changes to the protocol and the trial was stopped early. Protocol changes and details related to stopping the trial are reported according to the CONSERVE (CONSORT and SPIRIT Extension for RCTs Revised in Extenuating Circumstances) extension to the Consolidated Standards of Reporting Trials checklist. Protocol changes were approved by Health Canada as clinical trial amendments.

In September 2020, before participants were enrolled, we updated our sample size based on the results of a similar outpatient covid-19 trial in which our group had participated. That trial showed about 65\% symptom resolution by day 7 and defined the primary outcome of the trial as the proportion of participants with resolution of respiratory symptoms by day 7 .

In October 2020, as some regions were able to provide same day shipping, inclusion criteria were broadened to extend the onset of symptom duration from five to six days, only if the study drug could be delivered on the same day as enrolment. In our original protocol we included people who had had symptoms for five or fewer days but permitted next day shipping, effectively including some people with symptoms for six days. The study was run before the major roll out of the vaccination campaign in Canada. On 28 January 2021, the investigators decided to exclude vaccinated participants from the trial as they were hypothesised to have a reduced symptom burden and faster resolution of symptoms. No vaccinated participants were included in the trial.

At the end of May 2021, rates of vaccination in Canada were increasing rapidly and case numbers were declining noticeably. In the first two weeks of June, enrolment dropped off substantially from a peak of 22 participants each week to fewer than two participants each week across the country. A meeting with the data safety and monitoring board was held with 215 participants enrolled (203 in the modified intentionto-treat population) and without unblinding, as we noted that overall symptom resolution at day 7 was about 37\% compared with the projected 65\%. This was thought to be related to enrichment of participants with respiratory symptoms at enrolment. It was also noted that the STOIC trial (Inhaled Budesonide in the Treatment of Early COVID-19) had been reported and that budesonide was beginning to appear in provincial outpatient guidelines. Based on the drop off in newly diagnosed patients as we reached the summer months, and with the projected further increase in vaccination rates, we determined that we would be unable to recruit the intended sample size or to recruit to a larger sample size required to meet the intended effect size. With the permission of the data safety monitoring board, the study was terminated early for expected futility to meet total enrolment.

\section{Participants}

We included adults aged 18 years and older who had polymerase chain reaction confirmed covid-19 at 
enrolment with at least one of the symptoms of fever, cough (wet or dry), or shortness of breath (including dyspnoea, chest congestion, or chest tightness as synonyms). People were excluded if they were admitted to hospital, had only non-respiratory symptoms (eg, nasal congestion, myalgias, or gastrointestinal symptoms), had already been prescribed an inhaled steroid, or met other exclusion criteria (see supplementary appendix and trial protocol). All participants provided consent online.

\section{Randomisation and masking}

Randomisation was done centrally at the research pharmacy of the McGill University Health Centre in Montreal, Canada. The trial statistician generated a permuted block randomisation sequence using variably sized blocks of 2, 4, 6, and 8, with stratification according to sex. An unblinded research assistant sequentially assigned participants. The assignments were concealed from investigators and participants; only pharmacies and a central research assistant had access to the treatment allocation.

\section{Procedures}

Inhaled and intranasal ciclesonide were dispensed and shipped overnight to participants by commercial courier-except for participants who were enrolled on day 6 of their symptoms, who received the product the same day. The recommended dose range of inhaled ciclesonide for asthma is $100-800 \mu \mathrm{g} / \mathrm{day},{ }^{7}$ and 200 $\mu \mathrm{g} /$ day of the intranasal form is recommended for allergic rhinitis. As the specific dose of ciclesonide for the treatment of covid-19 was unknown, we gave a higher total daily dose (1200 $\mu \mathrm{g}$ divided twice daily) along with the usual dose of intranasal ciclesonide $(200 \mu \mathrm{g} /$ day) for 14 days. The control group received a saline placebo metered dose inhaler and intranasal saline of a similar appearance to ciclesonide at the same dosing schedule.

\section{Outcomes}

Participants self-reported outcome data for the 14 days after enrolment, including improvement in covid-19 related respiratory symptoms, other covid-19 symptoms, adherence to the trial intervention, side effects, and hospital admissions. Details of trial conduct are provided in the protocol and statistical analysis plan in the supplementary file.

We prespecified the primary outcome as the resolution of self-reported fever and all respiratory symptoms at day 7 of treatment. Respiratory symptoms included cough (wet or dry) or dyspnoea (which included the description of shortness of breath, chest congestion, or chest tightness as synonyms).

Key secondary outcomes included hospitaladmission or death with covid-19. Other secondary outcomes include evaluation of the primary outcome at day 14 , improvement in overall feeling (self-reported feeling much or very much better) by days 7 and 14, resolution of dyspnoea (defined as the absence of shortness of breath, chest tightness, or chest congestion) in the subset who reported a dyspnoea equivalent at baseline on days 7 and 14, improvement in cough at days 7 and 14 (defined as a 2 point decrease or a decrease to 0 on a visual analogue scale that ranged from 0 for no symptoms to 10 for severe symptoms) in those who had cough at baseline, improvement in shortness of breath as measured by the PROMIS (patient reported outcomes measurement information system) dyspnoea score, ${ }^{8}$ in sleep as measured by the PROMIS sleep disturbance score $4 \mathrm{a},{ }^{9}$ and anxiety as measured by the PROMIS emotional distress anxiety score $7 \mathrm{a}$ (with meaningful improvement defined as a $\geq 3$ point change on the T score). ${ }^{10}$

On each survey we collected adverse events using a combination of open ended free text questions about perceived side effects (which were categorised thematically) as well as specific questions about the presence of thrush, throat irritation, voice change, wheeze, nausea, and headache. Participants were also able to email or phone the study investigators if they developed worsening symptoms of covid-19 or possible side effects from the study drug, and these events were recorded.

\section{Statistical analysis}

Based on a previous covid-19 treatment trial with hydroxychloroquine, which was conducted in a similar population and used patient reported outcomes, ${ }^{11}$ we anticipated that symptoms would resolve in $65 \%$ of participants by day 7 of treatment. Using Fisher's exact method with a 15\% absolute risk to increase the proportion with resolution of symptoms to $80 \%$, a two sided $\alpha$ of 0.05 , and $80 \%$ power, and accounting for up to a $15 \%$ dropout rate, we estimated that 318 participants (159 in each group) would need to be enrolled.

\section{Interim analyses}

An independent data safety monitoring board reviewed the data after $50 \%$ of participants had been enrolled. No stopping criteria were provided, and the trial continued. A second meeting of the data safety monitoring board was held at the end of May in 2021, which led to the trial stopping early.

\section{Analysis}

TCL and AB analysed the data blinded to intervention group. We used binomial regression with an identity link to calculate risk differences for all proportions, which were adjusted for stratification by sex. ${ }^{12}$ All analyses were conducted with STATA software version 17 according to the modified intention-to-treat principle, with two sided type I error with an $\alpha$ of 0.05 . All risks and risk differences are presented as percentages. Participants were included in the modified intentionto-treat analysis if they received the shipped study drug, took at least one dose, and completed at least one follow-up survey. To avoid problems related to multiple comparisons, we present $\mathrm{P}$ values only for the primary outcome. For participants with missing survey data, we carried forward their last day's survey response. 
We also conducted a per protocol analysis, which excluded participants who were non-adherent to the study protocol (see supplementary file for definition). Prespecified subgroups included age older and younger than 40 years, sex, day of treatment initiation (day 1,2 , or $3 v$ day 4,5 , or 6 ), smoking status, ethnicity (white $v$ other), number of baseline symptoms of fever, cough, or dyspnoea $(1,2$, or 3$)$, and before and after the emergence of the alpha variant (March 2021).

\section{Patient and public involvement}

No patients or members of the public were involved in this research. Owing to the covid-19 pandemic and the rapidity with which the trial was started we did not involve members of the public while our trial was being implemented. We used the validated PROMIS patient reported outcome scores, which have been developed with patients with funding from the National Institutes of Health.

\section{Results}

Enrolment to the trial began on 15 September 2020 in Quebec, 9 February 2021 in Ontario, and 22 March 2021 in British Columbia. The last participants were recruited on 8 June 2021.

Overall, 215 participants were randomised, and the modified intention-to-treat population included 203 adults with respiratory symptoms or fever. Participants were assigned to either inhaled and intranasal ciclesonide $(n=105$; excluding two dropouts and one loss to follow-up) or inhaled and intranasal saline placebo $(\mathrm{n}=98$; excluding three dropouts and six losses to follow-up) (fig 1). The median age of participants was 35 years (interquartile range 2747 years $)$ and $54 \%(n=109)$ were women. Table 1 shows the personal and clinical characteristics of the participants. A total of $20 \%(n=41)$ reported a chronic health condition and only $7 \%(n=15)$ were smokers. Of the mandatory symptoms for study inclusion, cough was the most prevalent $(86 \% ; n=175)$, followed by dyspnoea, shortness of breath, chest tightness, or chest congestion ( $50 \% ; n=101)$ and fever $(46 \% ; n=93)$.

Fever and respiratory symptoms had resolved in 37\% $(n=76)$ of participants by day 7 . The proportion with resolved symptoms by day 7 did not differ significantly between the intervention group (42/105, 40\%) and control group (34/98, 35\%) (table 2). The adjusted risk difference was 5.5\% (95\% confidence interval $-7.8 \%$ to $18.8 \%$ ). The per protocol analysis was comparable, with an adjusted risk difference of $3.4 \%(-10.4 \%$ to $17.1 \%)$.

The proportion of participants with resolved symptoms at day 14 also did not differ significantly between the two groups, with $66 \%$ (69/105) showing resolution of symptoms by day 14 in the ciclesonide group compared with $58 \%$ (57/98) in the placebo group, with an adjusted risk difference of 7.5\% (95\% confidence interval $-5.9 \%$ to $20.8 \%$ ). Six participants in the ciclesonide group and three in the placebo group were admitted to hospital. No deaths occurred. The supplementary file presents the subgroup analyses along with the results of the PROMIS sleep, anxiety, and dyspnoea questionnaires, which did not detect statistically significant differences between groups.

Overall adherence was high-94\% of participants in the ciclesonide group (99/105) and 93\% in the placebo group (91/98) took the study drug on most survey days or at least until symptom resolution. Side effects were reported in 22\% (23/105) of participants in the ciclesonide group and $15 \%(15 / 98)$ in the placebo group (table 3 ). The most common reported side effects were headache and nausea, and these were more common in the treatment group. Overall, blinding was well maintained (see supplementary file).

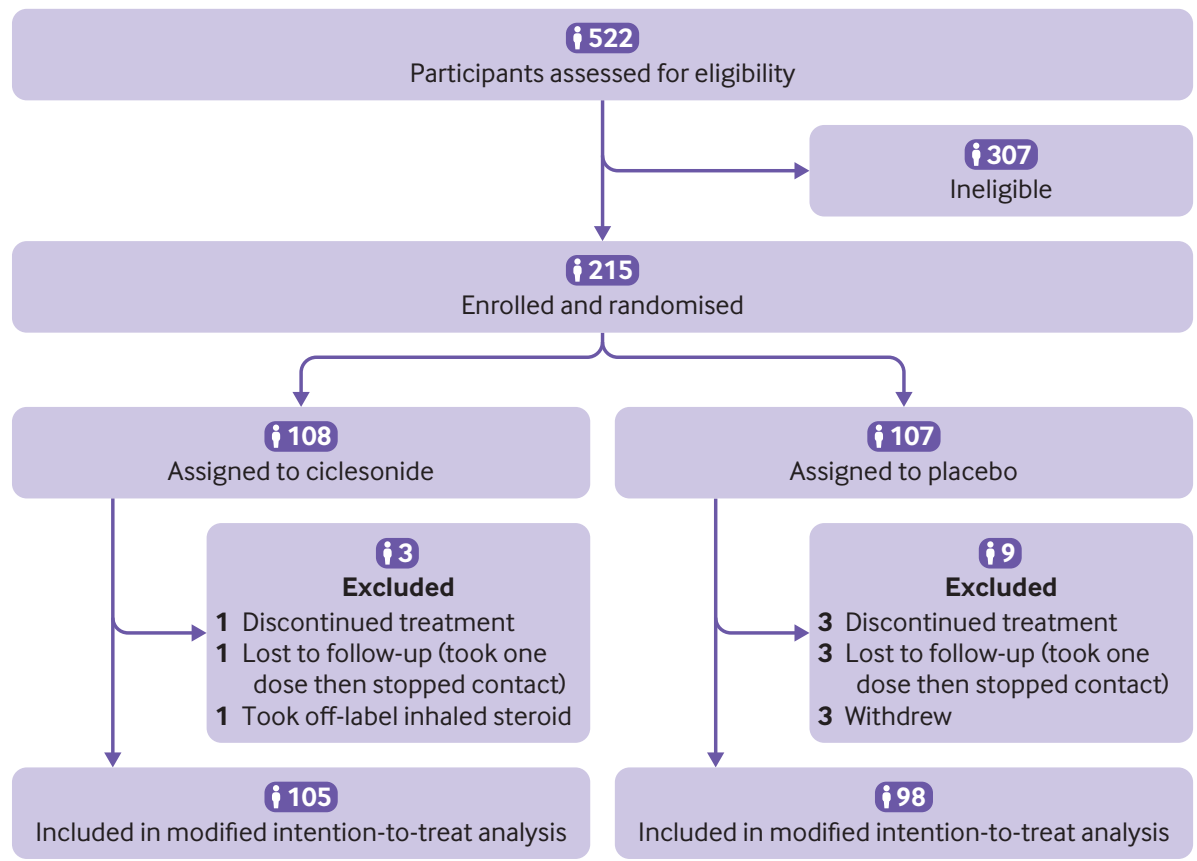

Fig 1 | Flow of participants through study 


\begin{tabular}{|c|c|c|}
\hline Characteristics & Placebo* $(n=98)$ & Ciclesonidet $(n=105)$ \\
\hline \multicolumn{3}{|l|}{ Personal } \\
\hline Median (IQR) age (years) & $35(27-45)$ & $35(27-47)$ \\
\hline Women & $55(56)$ & $54(51)$ \\
\hline Current smoker & $5(5)$ & $10(10)$ \\
\hline \multicolumn{3}{|l|}{ Ethnicity: } \\
\hline African Canadian & $2(2)$ & $3(3)$ \\
\hline Asian & $16(16)$ & $18(17)$ \\
\hline White & $58(59)$ & $65(62)$ \\
\hline Hispanic or Latino & $7(7)$ & $6(6)$ \\
\hline Middle Eastern & $12(12)$ & $6(6)$ \\
\hline South Asian & $6(6)$ & $6(6)$ \\
\hline Other & $2(2)$ & $3(3)$ \\
\hline \multicolumn{3}{|l|}{ Province: } \\
\hline British Columbia & $51(52)$ & $51(49)$ \\
\hline Ontario & $13(13)$ & $19(18)$ \\
\hline Quebec & $34(35)$ & $35(33)$ \\
\hline \multicolumn{3}{|l|}{ Symptoms: } \\
\hline Median (IQR) days of illness before screening & $3(2-4)$ & $3(2-4)$ \\
\hline Median (IQR) overall VAS (cm) & $5.0(3.6-5.9)$ & $5.0(3.2-5.8)$ \\
\hline Fever & $45(46)$ & $48(46)$ \\
\hline Cough & $86(88)$ & $89(85)$ \\
\hline Shortness of breath & $25(26)$ & $32(30)$ \\
\hline Chest tightness or chest congestion & $38(39)$ & $46(44)$ \\
\hline Dyspnoea $\neq$ & $49(50)$ & $53(50)$ \\
\hline Anosmia & $26(27)$ & $15(14)$ \\
\hline Loss of taste & $26(27)$ & $16(15)$ \\
\hline Sore throat & $41(42)$ & $39(37)$ \\
\hline Runny nose & $39(40)$ & $30(29)$ \\
\hline Sinus congestion & $50(51)$ & $51(49)$ \\
\hline Fatigue & $75(77)$ & $77(73)$ \\
\hline Myalgia & $58(59)$ & $66(63)$ \\
\hline Diarrhoea & $22(22)$ & $17(16)$ \\
\hline \multicolumn{3}{|l|}{ Health and comorbidities } \\
\hline \multicolumn{3}{|l|}{ Overall self-reported health: } \\
\hline Very good & $44(45)$ & $54(51)$ \\
\hline Good & $45(46)$ & $41(39)$ \\
\hline Moderate & $8(8)$ & $8(8)$ \\
\hline Very bad & $1(1)$ & $0(0)$ \\
\hline \multicolumn{3}{|l|}{ Comorbidities: } \\
\hline None & $76(78)$ & $86(82)$ \\
\hline Active cancer & $1(1)$ & $1(1)$ \\
\hline Asthma (no treatment) & $6(6)$ & $4(4)$ \\
\hline Diabetes mellitus & $4(4)$ & $1(1)$ \\
\hline Hypertension & $5(5)$ & $7(7)$ \\
\hline Ischaemic heart disease & $1(1)$ & $0(0)$ \\
\hline \multicolumn{3}{|c|}{$\begin{array}{l}\text { IQR=interquartile range; VAS=visual analogue scale. } \\
\text { Data represent patients from modified intention-to-treat population (those who took at least one dose of } \\
\text { study drug and completed at least one follow-up questionnaire related to symptom resolution compared with } \\
\text { baseline). } \\
\text { *Metered dose inhaler and nasal saline placebo. } \\
\text { tInhaled and intranasal. } \\
\text { fReported either shortness of breath or chest tightness or chest congestion. }\end{array}$} \\
\hline
\end{tabular}

\section{Discussion}

In this randomised double blind placebo controlled trial of intranasal and inhaled ciclesonide for the early treatment of covid-19, we were unable to detect a statistically significant improvement in respiratory symptoms of at day 7. Participants in this study were young and healthy and had a high burden of symptomatic respiratory disease. Most had a cough, and many had shortness of breath, chest congestion, and chest tightness. Adherence to the study drug was excellent, and blinding was adequately maintained. For the most part, treatment was initiated early in the course of the disease (median time to enrolment three days after the development of symptoms) and despite receiving a high dose of inhaled and intranasal ciclesonide, participants were not more likely to show symptom resolution by day 7 or 14 than those who received placebo.

\section{Comparison with other studies}

Two other randomised controlled trials of inhaled corticosteroids have been conducted. STOIC (Steroids in COVID-19) was an open label trial that compared inhaled budesonide with no budesonide in 146 adults with cough and with fever or anosmia, or both. ${ }^{13}$ The primary outcome was a composite of visits for urgent care, emergency department visits, and hospital admission. In a modified intention-to-treat analysis $(n=139)$, the primary outcome occurred in $11(15 \%)$ participants in the usual care group and two (3\%) in the budesonide group (difference in proportions 0.12 , 95\% confidence interval 0.03 to $0.21 ; \mathrm{P}=0.009$ ). Clinical recovery was also one day shorter in the budesonide group than usual care group (median 7 days (95\% confidence interval 6 to 9 days) $v 8$ days ( 7 to 11 days); log rank test $\mathrm{P}=0.007)$. The lack of placebo and the lack of blinding of study nurses, however, might have led to bias in care seeking behaviour in the usual care arm. This is especially relevant as inhaled drugs have been shown to have substantial placebo effects in respiratory diseases. ${ }^{14}$ The inclusion of urgent care visits (as opposed to hospital admissions) could have exacerbated this bias.

A second open label trial, the Platform Randomised Trial of Treatments in the Community for Epidemic and Pandemic Illnesses (PRINCIPLE) study ${ }^{15}$ also suggested a possible effect of inhaled budesonide, with a benefit in time to first self-reported recovery of 2.94 days ( $95 \%$ bayesian credible interval 1.19 to 5.12 days) (11.8 days (10.0 to 14.1 days) in the budesonide group $v 14.7$ days (12.3 to 18.0 days) in the usual care group; hazard ratio 1.21 (95\% bayesian credible interval 1.08 to 1.36)). The observed effect on symptom resolution was equally apparent among participants aged 50 to 65 years with comorbidities, and in people older than 65 years. Although our study was unable to detect a statistically significant effect on symptom resolution, we mainly enrolled younger, healthier participants. Similar to STOIC, a crucial limitation of the PRINCIPLE trial was a lack of placebo control such that the comparison of inhalers with usual care rendered it prone to the same potential for placebo effect and bias in healthcare seeking behaviour as the STOIC trial.

\section{Strengths and limitations of this study}

Our study has several strengths. It was placebo controlled, minimising bias; we explored adjunctive intranasal corticosteroids that directly deliver drugs to the nasal epithelium, the site of early viral replication; we selected the corticosteroid ciclesonide, which has in vitro antiviral effects ${ }^{6}$ and known antiinflammatory effects; and we studied a high dose to minimise the chance of a false negative study result 


\begin{tabular}{|c|c|c|c|}
\hline Outcomes & Placebo* $(n=98)$ & Ciclesonidet $(n=105)$ & Adjusted risk difference $\neq, \%(95 \% \mathrm{Cl})$ \\
\hline \multicolumn{4}{|l|}{ Primary outcome on day $7 \S$} \\
\hline Modified intention to treat & $34(35)$ & $42(40)$ & $5.5(-7.8$ to 18.8$)$ \\
\hline Per protocol & $32(35) ;(n=91)$ & $38(38) ;(n=99)$ & $3.4(-10.4$ to 17.1$)$ \\
\hline \multicolumn{4}{|l|}{ Secondary outcomes } \\
\hline Primary outcome on day 14 & $57(58)$ & $69(66)$ & $7.5(-5.9$ to 20.8$)$ \\
\hline \multicolumn{4}{|l|}{ Overall feeling of improvement: } \\
\hline Day 7 & $74(76)$ & $77(73)$ & $-2.1(-14.2$ to 9.9$)$ \\
\hline Day 14 & $91(93)$ & $95(90)$ & $-2.4(-10.0$ to 5.1$)$ \\
\hline \multicolumn{4}{|l|}{ Resolution of symptoms: } \\
\hline Day 7 & $22(22)$ & $25(24)$ & $0.8(-10.6$ to 12.2$)$ \\
\hline Day 14 & $44(45)$ & $57(54)$ & $9.1(-4.6$ to 22.8$)$ \\
\hline Admission to hospital: day 14 & $3(3)$ & $6(6)$ & $2.3(-3.0$ to 7.6$)$ \\
\hline \multicolumn{4}{|l|}{ Mortality: } \\
\hline Day 14 & $0(0)$ & $0(0)$ & - \\
\hline Day 29 & $0(0)$ & $0(0)$ & - \\
\hline Improvement in coughף: & $(n=86)$ & $(n=89)$ & \\
\hline Day 7 & $54(63)$ & $57(64)$ & $1.2(-12.9$ to 15.3$)$ \\
\hline Day 14 & $72(84)$ & $74(83)$ & $-0.9(-11.9$ to 10.1$)$ \\
\hline Resolution of dyspnoea**: & $(n=49)$ & $(n=53)$ & \\
\hline Day 7 & $27(55)$ & $38(71)$ & $15.8(-2.4$ to 34.0$)$ \\
\hline Day 14 & $40(82)$ & $45(85)$ & $3.9(-10.7$ to 18.5$)$ \\
\hline \multicolumn{4}{|c|}{$\begin{array}{l}\text { Data represent patients from modified intention-to-treat population (those who took at least one dose of study drug and completed at least one follow-up } \\
\text { questionnaire related to symptom resolution compared with baseline). } \\
\text { *Metered dose inhaler and nasal saline placebo. } \\
\text { tInhaled and intranasal. } \\
\text { fAdjusted for stratification on sex. } \\
\text { SResolution of fever and all respiratory symptoms by day } 7 \text {. } \\
\text { १Analysis limited to patients who reported dry or wet cough at baseline. } \\
\text { **Dyspnoea defined as shortness of breath, chest tightness, or chest congestion. Analysis limited to participants who reported dyspnoea at baseline. }\end{array}$} \\
\hline
\end{tabular}

due to inadequate exposure. Finally, we recruited participants with important respiratory symptoms, comprising the population most likely to benefit from inhaled steroids. Moreover, participants were enrolled early in the course of their disease (within an average of three days), which is probably similar to what is achievable in a real world setting when factoring in delays to seeking medical attention.

Despite these strengths, our study has some limitations. We were unable to recruit the intended sample size given a rapid decline in cases of covid-19 in Canada following increases in vaccination. However, we recruited more participants than the STOIC trial,

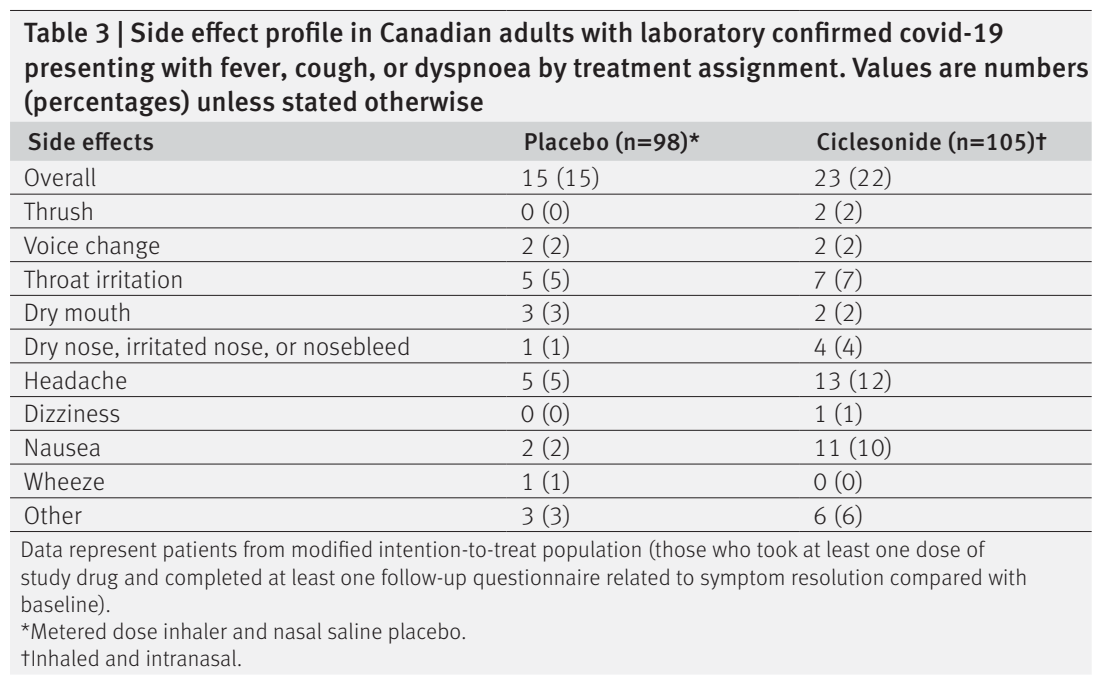

which found a difference in effect. In addition, we did not enrich the study population for older high risk people and cannot exclude a possible benefit in earlier symptom resolution and prevention of clinical deterioration in this group. That said, the overall rate of hospital admissions in our study was similar to those of other outpatient trials, ${ }^{11}$ including one that enriched for higher risk participants. ${ }^{16}$ We elected to examine the primary outcome at seven days after enrolment based on previous data that suggested up to $65 \%$ resolution over this timeframe. ${ }^{11}$ Although the overall resolution of symptoms by day 7 after enrolment was lower than expected, no appreciable difference was found in the secondary outcome at day 14 . It is important to mention that vaccinated participants were excluded from this trial. It was assumed vaccination would decrease the burden and duration of symptoms and risk of severe complications, which were our primary and secondary outcomes. It remains to be determined whether inhaled corticosteroids can reduce symptoms in vaccinated populations, but we expect that any observed effect would be smaller than in unvaccinated people.

As our trial was stopped prematurely, we might have been underpowered to show a small benefit of ciclesonide on early symptom resolution. Recently, another placebo controlled study (400 participants) of ciclesonide was published as a preprint and also failed to show any effect on symptom resolution. ${ }^{17}$ Although our study found an absolute risk reduction of 5.5\%, the confidence interval was wide. A meta-analysis might further elucidate whether this relatively inexpensive 
and generally well tolerated treatment shows clinical benefit.

\section{Conclusion}

Inhaled corticosteroids might improve the resolution of covid19 symptoms among older high risk adults, and younger healthier populations could be less likely to benefit. In vitro data suggest that ciclesonide possesses antiviral activity against SARS-CoV-2. However, our placebo controlled randomised trial failed to show statistically significant improvement in symptoms among healthy, young participants who presented with prominent respiratory symptoms. Based on our findings, we suggest that future studies should enrich for patients reporting baseline shortness of breath and examine outcomes to day 14, given the prolonged time to symptom resolution that we, and others, have observed. Open label studies should be avoided, and additional placebo controlled studies should examine the effect of different classes of inhaled corticosteroids.

\section{AUTHOR AFFILIATIONS}

${ }^{1}$ Department of Medicine, Division of Respirology, McGill University Health Centre, Montreal, Quebec, Canada

${ }^{2}$ Research Institute of the McGill University Health Centre, Montreal, Quebec, Canada

${ }^{3}$ Division of Infectious Diseases, University of British Columbia, Vancouver, British Columbia, Canada

${ }^{4}$ Department of Medicine, Division of Infectious Diseases, Sunnybrook Health Sciences Centre, University of Toronto, Toronto, Ontario, Canada

${ }^{5}$ Clinical Practice Assessment Unit, Department of Medicine, Montreal, Quebec, Canada

${ }^{6}$ Department of Medicine, Division of Clinical Epidemiology, Department of Medicine, McGill University Health Centre, Montreal, Quebec, Canada

${ }^{7}$ Department of Medicine, Division of Infectious Diseases, McGill University Health Centre, Montreal, Quebec, Canada

${ }^{8}$ Department of Medicine, Division of General Internal Medicine, McGill University Health Centre, Montreal, Quebec, Canada

We thank the Vancouver Coastal Health Research Institute, particularly Christopher Mah, Joleen Wright, and Stephania Manusha, as well as Amina Moustaqim-Barrette from the Public Health Surveillance Unit of Vancouver Coastal Health $(\mathrm{VCH})$, for providing the $\mathrm{VCH}$ regional list for participant recruitment. The investigators thank all members of the CONTAIN Team

Inhaled and Intranasal Ciclesonide for the Treatment of COVID-19 in Nonhospitalized Adults (CONTAIN) team: McGill University Health Centre-Annie-Claude Jalbert, drafting of Health Canada protocol, Charlotte Besson, research coordination, Kristen Moran, research coordination, Alek Lefevbre, database co-design and maintenance, Kathleen Normandin and the Research Pharmacy of the McGill University Health Centre, Sarah Elsayed, research coordination, Rola Hamed, research coordination, and Francine Noel, research coordination

Data safety and monitoring board members-Dick Menzies, Faiz Khan, and Sandra Dial

University of British Columbia-Vincent Hou, research assistant, Gale Ladua, research assistant, Dilpreet Bharaj, research assistant, and Alan Low, James Chow, and Judy Lee, BioPro Biologics Pharmacy

Sunnybrook Health Sciences Centre-Jose Estrada-Codecido Contributors: NE, BMS, SJB, AB, JGM, TCL, and EGM conceived the study. NE, SJB, $A B$, and EGM were responsible for the methods. NE, $\mathrm{KM}, \mathrm{CB}$, and $\mathrm{TCL}$ validated the data. TCL and $\mathrm{AB}$ performed the main analysis. NE, SB, ND, AC, SD, CB, KM, LYS, EGM were responsible for study conduct NE, SB, ND, AC, TCL, and EGM were responsible for financing. NE, KM, and TCL curated the data. NE, EGM, and TCL wrote the original draft of the manuscript. All authors wrote, reviewed, and edited the manuscript. NE, EGM, and JGM supervised the study. NE,
$\mathrm{KM}$, and EGM were responsible for administration of the project. NE and EGM are the guarantors. The corresponding author attests that all listed authors meet authorship criteria and that no others meeting the criteria have been omitted.

Funding: The placebo metered dose inhaler was donated by GlaxoSmithKline. Nasal saline, and spacers for the inhalers were purchased by NE. The study drug was donated by COVIS Pharma. Glaxo Smith Kline and COVIS Pharma provided no salary support for investigators or study staff and did not contribute to the study design, analysis, or manuscript preparation. The study was funded by the McGill University Health Centre Foundation and the McGill Interdisciplinary Initiative in Infection and Immunity. The funders had no role in considering the study design or in the collection, analysis, interpretation of data, writing of the report, or decision to submit the article for publication.

Competing interests: All authors have completed the ICMJE uniform disclosure form at www.icmje.org/disclosure-of-interest/ and declare: NE reports grants from Fonds de Recherche du Québec en Santé, grants from Rossy Cancer Network, non-financial support from COVIS Pharma (study drug donation), advisory board fees from Glaxo Smith Kline, advisory board fees from Astra Zeneca, grants from Canadian Institute of Health Research, grants from MEDTEQ, outside the submitted work. SB reports personal fees from Verity Pharma and from Merck, outside the submitted work. All remaining authors declare no support from any organisation for the submitted; no financial relationships with any organisations that might have an interest in the submitted work in the previous three years; no other relationships or activities that could appear to have influenced the submitted work.

Ethical approval: The study was approved by the research ethics boards of McGill University Health Centre (ID 2021-6696), Sunnybrooke Health Sciences Centre, and the University of British Colombia. The clinical trial was also submitted to Health Canada (control No 239123), which provided a no objection letter for the trial duration.

Data sharing: Deidentified participant data will be made available six months after publication of this study for 12 months by contacting TCL at todd.lee@mcgill.ca for a specified purpose through a data sharing agreement, following approval of the corresponding author (NE) and with investigator support. A data dictionary will be provided. No additional data shall be provided.

The lead author affirms that the manuscript is an honest, accurate, and transparent account of the study being reported; no important aspects of the study have been omitted; any discrepancies from the study as originally planned (and, if relevant, registered) have been explained.

Dissemination to participants and related patient and public communities: Results will be shared on the website of the trial (www.contain-covid19.com) for research participants and the public, and disseminated by the communications office of the McGill University Health Centre, Sunnybrooke Health Sciences Centre, and the University of British Colombia. It will also be shared by study investigators through social media platforms.

Provenance and peer review: Not commissioned; externally peer reviewed.

This is an Open Access article distributed in accordance with the Creative Commons Attribution Non Commercial (CC BY-NC 4.0) license, which permits others to distribute, remix, adapt, build upon this work non-commercially, and license their derivative works on different terms, provided the original work is properly cited and the use is noncommercial. See: http://creativecommons.org/licenses/by-nc/4.0/.

1 Horby P, Lim WS, Emberson IR, et al, RECOVERY Collaborative Group. Dexamethasone in Hospitalized Patients with Covid-19. N Engl J Med 2021;384:693-704. doi:10.1056/NEJMoa2021436

2 Lawler PR, Goligher EC, Berger IS, et al, ATTACC Investigators, ACTIV-4a Investigators, REMAP-CAP Investigators. Therapeutic Anticoagulation with Heparin in Noncritically Ill Patients with Covid-19. N Engl J Med 2021;385:790-802

3 Gordon AC, Mouncey PR, Al-Beidh F, et al, REMAP-CAP Investigators. Interleukin-6 Receptor Antagonists in Critically III Patients with Covid-19. N Engl J Med 2021;384:1491-502. doi:10.1056/ NEIMoa2100433

4 Weinreich DM, Sivapalasingam S, Norton T, et al, Trial Investigators. REGN-COV2, a Neutralizing Antibody Cocktail, in Outpatients with Covid-19. N Engl J Med 2021;384:238-51. doi:10.1056/ NEIMoa2035002

5 Jeon S, Ko M, Lee J, et al. Identification of Antiviral Drug Candidates against SARS-CoV-2 from FDA-Approved Drugs. Antimicrob Agents Chemother 2020;64:e00819-20, doi:10.1128/AAC.00819-20 
6 Matsuyama S, Kawase M, Nao N, et al. The Inhaled Steroid Ciclesonide Blocks SARS-CoV-2 RNA Replication by Targeting the Viral Replication-Transcription Complex in Cultured Cells. Virol 2020;95:e01648-20. doi:10.1128/JVI.01648-20

7 Health Canada. Product monograph. ALVESCO®. 2021. ttps://pdf. hres.ca/dpd pm/00060608.PDF

8 PROMIS. DYSPNEA: A brief guide to the PROMIS@ Dyspnea instruments. Accessed July 23, 2021. http://www.healthmeasures. net/images/PROMIS/manuals/PROMIS_Dyspnea_Scoring_ Manual.pdf

9 Yu L, Buysse DJ, Germain A, et al. Development of short forms from the PROMISTM sleep disturbance and Sleep-Related Impairment item banks. Behav Sleep Med 2011;10:6-24. doi:10.1080/15402002.2 012.636266

10 Pilkonis PA, Choi SW, Reise SP, Stover AM, Riley WT, Cella D, PROMIS Cooperative Group. Item banks for measuring emotional distress from the Patient-Reported Outcomes Measurement Information System (PROMIS $®$ ): depression, anxiety, and anger Assessment 2011;18:263-83 doi:10.1177/1073191111411667

11 Skipper CP, Pastick KA, Engen NW, et al. Hydroxychloroquine in Nonhospitalized Adults With Early COVID-19: A Randomized Trial. Ann Intern Med 2020;173:623-31. doi:10.7326/M20-4207

12 Kahan BC, Morris TP. Reporting and analysis of trials using stratified randomisation in leading medical journals: review and reanalysis. BMJ 2012;345:e5840. doi:10.1136/bmj.e5840
13 Ramakrishnan S, Nicolau DVJr, Langford B, et al. Inhaled budesonide in the treatment of early COVID-19 (STOIC): a phase 2, open-label, randomised controlled trial. Lancet Respir Med 2021;9:763-72. doi:10.1016/S2213-2600(21)00160-0

14 Wechsler ME, Kelley JM, Boyd IOE, et al. Active albuterol or placebo, sham acupuncture, or no intervention in asthma. N Engl Med 2011;365:119-26. doi:10.1056/NEJMoa1103319

15 Yu LM, Bafadhel M, Dorward J, et al, PRINCIPLE Trial Collaborative Group. Inhaled budesonide for COVID-19 in people at high risk of complications in the community in the UK (PRINCIPLE) a randomised, controlled, open-label, adaptive platform trial. Lancet 2021;398:843-55. doi:10.1016/S0140-6736(21)01744-X

16 Tardif JC, Bouabdallaoui N, L'Allier PL, et al, COLCORONA Investigators. Colchicine for community-treated patients with COVID-19 (COLCORONA): a phase 3, randomised, double-blinded, adaptive, placebo-controlled, multicentre trial. Lancet Respir Med 2021;9:924-32. doi:10.1016/S2213-2600(21)00222-8

17 Clemency BM, Varughese R, Gonzalez-Rojas Y, Morse CG, Phipatanakul W, Koster DJ, Blaiss MS. A randomized controlled trial of inhaled ciclesonide for outpatient treatment of symptomatic COVID-19 infections.MedRxiv . 2021 doi:10.1101/2021.09.07.21261811

Supplementary file: additional information 